\title{
The Design and Research of the Sliding Mode Control of a New Chaotic System
}

\author{
Xuexiang Li \\ Zibo Vocational Institute, China \\ lixuexiang2000@163.com
}

\begin{abstract}
This thesis makes a research about the Sliding Mode Control of a new chaotic system. Taking advantage of PI switching manifold method, it projects a kind of sliding mode controller and fuzzy the switching function of sliding mode surface with membership function, then finally adopts the output of fuzzy controller. What's more, this method simultaneously makes full use of PI control as well as SMC based on the new area control error, choosing the integral sliding surface equation which makes the system enter into the state of sliding mode from the beginning. Then it turns into the PI control on the basis of new area control error. Making the system output track a given goal until the elimination of tracking error in the main theoretically proves the global stability of this sliding mode controller, and the numerical simulation experiment further shows its simplicity as well as availability, besides, makes sure the steady availability of the whole system.
\end{abstract}

Keywords: New chaotic system, PI, Sliding Mode Control

\section{Introduction}

Chaotic motion was first discovered in 1963 by an American meteorologist Lorenz when he studied regional microclimate and tried to solve model equation. In 1982, Holden found the chaotic attracter and Moon discovered it again in physical system in 1992. Later, in 2003, Liu and Chen put forward a new kind of chaotic system, which revealed complexed two and four vortexes in simulation experiment.

Recently, Yassen designs a linearity feedback controller and achieves the control of novel chaotic system equipped with the quality of particle stability; and Chang uses sliding mode control to realize the chaotic control of Rössler's system. On the basis of their representative analysis, this thesis projects a kind of sliding mode controller and theoretically proves its global stability, besides, the numerical simulation experiment further shows its simplicity as well as availability.

\section{The Design of Sliding Mode Controller}

Representatively, considering the following nonlinear system

$$
\dot{\boldsymbol{X}}=\boldsymbol{A X}+\boldsymbol{B F}(\boldsymbol{X})+\boldsymbol{C}
$$

Here $\boldsymbol{A} \in R^{n \times n}, \boldsymbol{B} \in R^{n \times m}, \boldsymbol{C} \in R^{n \times 1}, \boldsymbol{F}: R^{n} \rightarrow R^{m}(m \leq n), \boldsymbol{F}(\boldsymbol{X})$ is nonlinear mapping, $\boldsymbol{A} \boldsymbol{X}$ is linear segment, $\boldsymbol{B F}(\boldsymbol{X})$ is nonlinear segment, $\boldsymbol{X} \in R^{n \times 1}$ is state variety, $\boldsymbol{C}$ is a constant matrix. 
Make the equilibrium point of system (1) as $\overline{\boldsymbol{X}}=\left[\begin{array}{llll}\bar{x}_{1} & \bar{x}_{2} & \ldots & \bar{x}_{n}\end{array}\right]^{\mathrm{T}}$, the error between state variety and equilibrium point is

$$
\boldsymbol{E}=\left[\begin{array}{c}
\boldsymbol{E}_{1}(t) \\
\boldsymbol{E}_{2}(t) \\
\vdots \\
\boldsymbol{E}_{n}(t)
\end{array}\right]=\left[\begin{array}{c}
x_{1}-\bar{x}_{1} \\
x_{2}-\bar{x}_{2} \\
\vdots \\
x_{n}-\bar{x}_{n}
\end{array}\right] .
$$

The controlled form in system (1) is

$$
\begin{gathered}
\dot{\boldsymbol{X}}=\boldsymbol{A} \boldsymbol{X}+\boldsymbol{B F}(\boldsymbol{X})+\boldsymbol{C}+\boldsymbol{U}, \\
\boldsymbol{U}=\left[\begin{array}{c}
u_{1}(t) \\
u_{2}(t) \\
\vdots \\
u_{m}(t)
\end{array}\right],
\end{gathered}
$$

Here $\boldsymbol{U} \in R^{m \times 1}$ is control item, m means the number of nonlinear items in system (3). From (2) and (3) we can conclude that the error dynamic system is

$$
\dot{\boldsymbol{E}}=\dot{\boldsymbol{X}}-\dot{\overline{\boldsymbol{X}}}=\boldsymbol{A E}+\boldsymbol{B F ^ { \prime }}+\boldsymbol{B U},
$$

Here $\boldsymbol{F}^{\prime} \in R^{m \times 1}$ is the error of relevant nonlinear items in system (3). And our control objective is to design suitable sliding mode controller and meet the following equation

$$
\lim _{t \rightarrow \infty}\|\boldsymbol{E}(t)\|=\lim _{t \rightarrow \infty}\left\|\left[E_{1}(t) E_{2}(t) \cdots E_{n}(t)\right]^{T}\right\| \rightarrow 0 .
$$

Usually, the design of sliding mode controller consists of two parts. Firstly, it should choose a switching manifold and assure that

$$
\lim _{t \rightarrow \infty}\|E\| \rightarrow 0
$$

Then examine the controller again to make the error dynamic system (5) arrive at the switching manifold within limited time, along which it can march towards the equilibrium point [1]. And the key is to select the suitable as well as stable switching manifold and to examine the sliding mode controller for making the system approach the switching manifold as soon as possible. Basing on the theory mentioned above, we can define PI switching manifold as

$$
\boldsymbol{S}=\boldsymbol{C E}-\int_{0}^{t}(\boldsymbol{C A}+\boldsymbol{C B K}) \boldsymbol{E}(\tau) d \tau,
$$

Here $\boldsymbol{S}=\left[s_{1} s_{2} \ldots s_{m}\right]^{\mathrm{T}}, \boldsymbol{C} \in R^{m \times n}, \boldsymbol{K} \in R^{m \times n}$. Choose $\boldsymbol{C}$ and make it meet the condition of $\boldsymbol{C B} \neq 0$, that is to say, $\boldsymbol{C B}$ is nonsingular matrix. At the same time, choose $\boldsymbol{K}$ to make all the real parts of eigenvalue in matrix $\boldsymbol{A}+\boldsymbol{B} \boldsymbol{K}$ are negative numbers, so $\boldsymbol{A}+\boldsymbol{B} \boldsymbol{K}$ is 
steady. According to the theory of variable structure control, it should satisfy the following equation when error dynamic system (5) doing the sliding mode motion [2].

$$
\begin{gathered}
\boldsymbol{S}=\boldsymbol{C E}-\int_{0}^{t}(\boldsymbol{C A}+\boldsymbol{C B K}) \boldsymbol{E}(\tau) d \tau=0, \\
\dot{\boldsymbol{S}}=\boldsymbol{C} \dot{\boldsymbol{E}}-(\boldsymbol{C A}+\boldsymbol{C B K}) \boldsymbol{E}=0 .
\end{gathered}
$$

From equation (5) and (9)

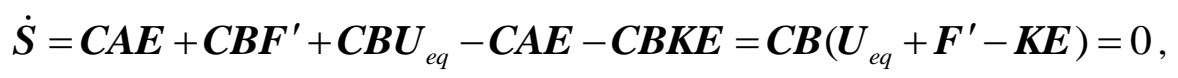

As $\boldsymbol{C B} \neq 0$, the equivalent control of sliding mode is

$$
\boldsymbol{U}_{e q}=\boldsymbol{K} \boldsymbol{E}-\boldsymbol{F}^{\prime}
$$

From equation (5) and (11)

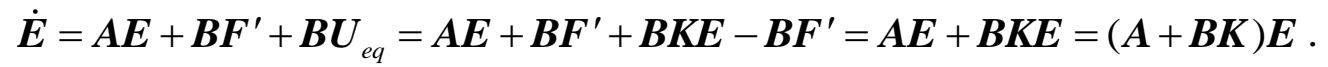

From (12) it can conclude that we can choose suitable $\boldsymbol{K}$ through pole assignment method to make sure that all the eigenvalue of matrix $\boldsymbol{A}+\boldsymbol{B} \boldsymbol{K}$ are negative numbers, in this way can ensure the steadiness of error dynamic system (5) on the switching manifold as well as $\lim _{t \rightarrow \infty}\|\boldsymbol{E}\|=0$

Next, we will design a relevant control rate to assure that the error dynamic system (5) arrive at the switching manifold within limited time and further achieve the sliding mode motion [3].

Lemma One If the condition of arrival in the following sliding mode established, the sliding mode motion will go into a stable situation step by step.

$$
\boldsymbol{S}^{\mathrm{T}}(t) \dot{\boldsymbol{S}}(t)<0 .
$$

Proof: Make the function of Lyapunov as

$$
V(t)=\frac{1}{2} \boldsymbol{S}^{\mathrm{T}}(t) \boldsymbol{S}(t)
$$

The derivative of $\mathrm{V}(\mathrm{t})$ is

$$
\dot{V}(t)=\boldsymbol{S}^{\mathrm{T}}(t) \dot{\boldsymbol{S}}(t) .
$$

According to Lyapunov's stability theory, if $\boldsymbol{S}^{\mathrm{T}}(t) \dot{\boldsymbol{S}}(t)<0$, the origin becomes gradually state point, thus $\lim _{t \rightarrow \infty} S(t)=0$. Proof finished [4-6].

In order to make sure the establishment of condition in Lemma One, relevant control rate is given by the following equation

$$
\boldsymbol{U}=-\lambda(\boldsymbol{C B})^{-1}\|\boldsymbol{C B}\|\left(\|\boldsymbol{K} \boldsymbol{E}\|+\left\|\boldsymbol{F}^{\prime}\right\|\right) \operatorname{sign}(\boldsymbol{S}),
$$

$$
\text { Here }^{\lambda>1}, \operatorname{sign}(\cdot) \text { is sign function, so if } S>0, \operatorname{sign}(S)=1 \text {; if } \boldsymbol{S}<0, \operatorname{sign}(\boldsymbol{S})=-1 \text {; }
$$


if $S=0, \operatorname{sign}(S)=0$. Then we will prove that the mentioned control rate can make the error dynamic system arrive at the switching manifold and finally achieve sliding mode motion.

Theorem one: The control rate $U$ given by (15) can make the arrival condition of sliding mode in (13) valid.

Proof: combing (5) and (15) with $\boldsymbol{S}^{\mathrm{T}}(t) \dot{\boldsymbol{S}}(t)$ :

$$
\begin{aligned}
& \boldsymbol{S}^{\mathrm{T}} \dot{\boldsymbol{S}}=\boldsymbol{S}^{\mathrm{T}}[\boldsymbol{C} \dot{\boldsymbol{E}}-\boldsymbol{C A} \boldsymbol{E}-\boldsymbol{C B K} \boldsymbol{E}]=\boldsymbol{S}^{\mathrm{T}}\left[\boldsymbol{C B} \boldsymbol{F}^{\prime}-\lambda\|\boldsymbol{C B}\|\left(\|\boldsymbol{K} \boldsymbol{E}\|+\left\|\boldsymbol{F}^{\prime}\right\|\right) \operatorname{sign}(\boldsymbol{S})-\boldsymbol{C B K} \boldsymbol{E}\right] \\
& \leq-\lambda\|\boldsymbol{C B}\|\left(\|\boldsymbol{K} \boldsymbol{E}\|+\left\|\boldsymbol{F}^{\prime}\right\|\right) \boldsymbol{S}^{\mathrm{T}} \operatorname{sign}(\boldsymbol{S})+\left(\left\|\boldsymbol{C B} \boldsymbol{F}^{\prime}\right\|+\|\boldsymbol{C B K E}\|\right)\|\boldsymbol{S}\| . \\
& { }_{\text {As }} S^{\mathrm{T}} \operatorname{sign}(S) \geq\|S\|, \lambda>1 \text {, so } \\
& \boldsymbol{S}^{\mathrm{T}} \dot{\boldsymbol{S}} \leq(1-\lambda)\left[\|\boldsymbol{C B}\|\left(\|\boldsymbol{K} \boldsymbol{E}\|+\left\|\boldsymbol{F}^{\prime}\right\|\right)\right]\|\boldsymbol{S}\|<0,
\end{aligned}
$$

The arrival condition of sliding mode founded. Proof finished.

\section{The Description of New Chaotic System and Its Sliding Mode Control}

The new chaotic system is:

$$
\left\{\begin{array}{l}
\dot{x}_{1}=a x_{1}-x_{2} x_{3} \\
\dot{x}_{2}=-b x_{2}+x_{1} x_{3}, \\
\dot{x}_{3}=-c x_{3}+x_{1} x_{2}
\end{array}\right.
$$

Here the controller parameter $a>0, b>0, c>0$. When $a=4.5, b=12, c=5$, system (18) becomes chaotic attractors with two vortexes. When $a=0.4, b=12, c=5$, system (18) turns into chaotic attractors with four vortexes [7-9]. Figure 1 shows the relevant chaotic attractors in system (18).

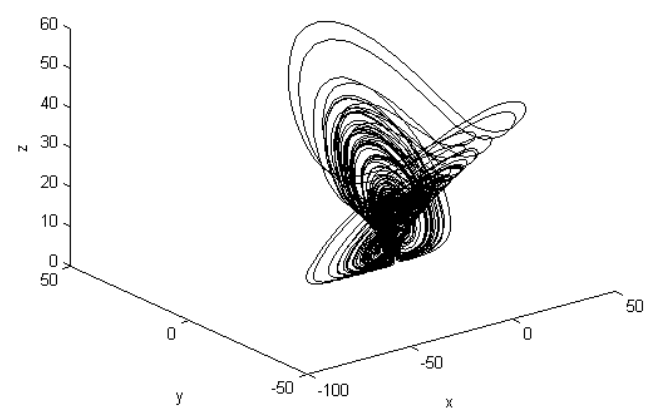

(a) When $a=4.5, b=12, c=5$, the chaotic attractors with two vortexes 


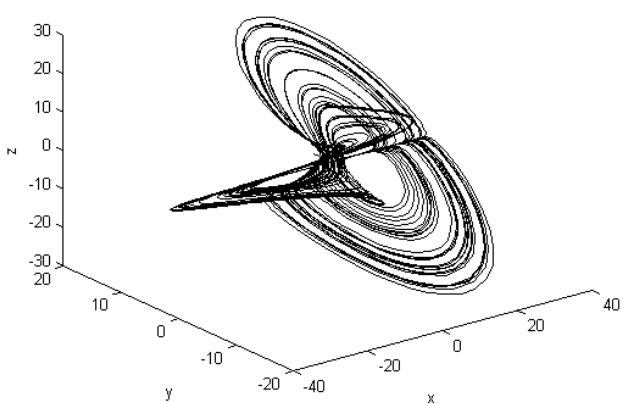

(b) When $a=0.4, b=12, c=5$, the chaotic attractors with four vortexes

\section{Figure 1. The chaotic attractors in system (18)}

Rewriting the system (18) with system (1)

$$
\dot{\boldsymbol{X}}=\boldsymbol{A} \boldsymbol{X}+\boldsymbol{B F}(\boldsymbol{X}),
$$

Here

$$
\boldsymbol{A}=\left[\begin{array}{ccr}
a & 0 & 0 \\
0 & -b & 0 \\
0 & 0 & -c
\end{array}\right], \boldsymbol{B}=\left[\begin{array}{lll}
1 & 0 & 0 \\
0 & 1 & 0 \\
0 & 0 & 1
\end{array}\right], \quad \dot{\boldsymbol{X}}=\left[\begin{array}{c}
\dot{x}_{1} \\
\dot{x}_{2} \\
\dot{x}_{3}
\end{array}\right], \quad \boldsymbol{X}=\left[\begin{array}{c}
x_{1} \\
x_{2} \\
x_{3}
\end{array}\right], \quad \boldsymbol{F}(\boldsymbol{X})=\left[\begin{array}{c}
-x_{2} x_{3} \\
x_{1} x_{3} \\
x_{1} x_{2}
\end{array}\right] .
$$

The controlled form of system (18)

$$
\dot{\boldsymbol{X}}=\boldsymbol{A} \boldsymbol{X}+\boldsymbol{B F}(\boldsymbol{X})+\boldsymbol{U},
$$

Here

$$
\boldsymbol{U}=\left[\begin{array}{l}
u_{1}(t) \\
u_{2}(t) \\
u_{3}(t)
\end{array}\right]
$$

is undetermined control item, error dynamic system (5) can be concretized as

$$
\dot{E}=\boldsymbol{A} \boldsymbol{E}+B F^{\prime}+B \boldsymbol{U},
$$

Here

$$
\boldsymbol{E}=\left[\begin{array}{l}
E_{1}(t) \\
E_{2}(t) \\
E_{3}(t)
\end{array}\right]=\left[\begin{array}{c}
x_{1}-\bar{x}_{1} \\
x_{2}-\bar{x}_{2} \\
x_{3}-\bar{x}_{3}
\end{array}\right], \quad \boldsymbol{F}^{\prime}=\left[\begin{array}{c}
-\left(x_{2} x_{3}-\bar{x}_{2} \bar{x}_{3}\right) \\
x_{1} x_{3}-\bar{x}_{1} \bar{x}_{3} \\
x_{1} x_{2}-\bar{x}_{1} \bar{x}_{2}
\end{array}\right]=\left[\begin{array}{c}
-E_{2} E_{3}-E_{2} \bar{x}_{3}-E_{3} \bar{x}_{2} \\
E_{1} E_{3}+E_{1} \bar{x}_{3}+E_{3} \bar{x}_{1} \\
E_{1} E_{2}+E_{1} \bar{x}_{2}+E_{2} \bar{x}_{1}
\end{array}\right] .
$$

In order to test the availability of controller (15), this thesis will regulate respectively the chaotic attractors with two or four vortexes, making them stabilize at the settled equilibrium point from random initial state within limited time. 


\section{Simulation Experiment}

\subsection{The control of chaotic attractor with two vortexes}

Make controller parameter $a=4.5, b=12, c=5$, and choose

$$
\boldsymbol{C}=\left[\begin{array}{lll}
1 & 0 & 0 \\
0 & 1 & 0 \\
0 & 0 & 1
\end{array}\right],
$$

When $\boldsymbol{C B} \neq 0$. Use pole assignment method to choose

$$
\boldsymbol{K}=\left[\begin{array}{lll}
-7.5 & 0 & 0 \\
0 & 10 & 0 \\
0 & 0 & 4
\end{array}\right]
$$

Make sure that all the real parts of eigenvalue in matrix $\boldsymbol{A}+\boldsymbol{B} \boldsymbol{K}$ are negative numbers. Then take constant $\lambda=1.6$ and meet the condition of $\lambda>1$.

$$
\begin{gathered}
\boldsymbol{S}=\boldsymbol{C} \boldsymbol{E}-\int_{0}^{t}(\boldsymbol{C} \boldsymbol{A}+\boldsymbol{C B} \boldsymbol{K}) \boldsymbol{E}(\tau) d \tau \Rightarrow\left\{\begin{array}{l}
s_{1}=E_{1}(\tau)-\int_{0}^{t}\left[\begin{array}{lll}
-3 & 0 & 0
\end{array}\right] \boldsymbol{E}(\tau) d \tau \\
s_{2}=E_{2}(\tau)-\int_{0}^{t}\left[\begin{array}{lll}
0 & -2 & 0
\end{array}\right] \boldsymbol{E}(\tau) d \tau \\
s_{3}=E_{3}(\tau)-\int_{0}^{t}\left[\begin{array}{lll}
0 & 0 & -1
\end{array}\right] \boldsymbol{E}(\tau) d \tau
\end{array}\right. \\
U=-\lambda(\boldsymbol{C B})^{-1}\|\boldsymbol{C B}\|(\|\boldsymbol{K} \boldsymbol{E}\|+\|\boldsymbol{F}\|) \operatorname{sign}(\boldsymbol{S})
\end{gathered}
$$

Adopt Runge-Kutta's method to solve system (20) and select time step $\tau=0.005$ (sec). The equiibrium point of system (18) is $\overline{\boldsymbol{X}}_{4}=\left[\begin{array}{lll}7.746 & 4.7434 & 7.3485\end{array}\right]^{\mathrm{T}}$. The initial value of system which stabilizes at $\overline{\boldsymbol{X}}_{0}$ points is $\boldsymbol{X}_{00}=\left[\begin{array}{lll}-6 & 1.7 & -8\end{array}\right]^{\mathrm{T}}$, the value at $\overline{\boldsymbol{X}}_{1}$ and $\overline{\boldsymbol{X}}_{2}$ point is $\boldsymbol{X}_{01}=\boldsymbol{X}_{02}=\left[\begin{array}{lll}3 & 5 & 8\end{array}\right]^{\mathrm{T}}$, and the value at $\overline{\boldsymbol{X}}_{3}$ and $\overline{\boldsymbol{X}}_{4}$ point ${ }_{\text {is }} \boldsymbol{X}_{03}=\boldsymbol{X}_{04}=\left[\begin{array}{lll}-3 & -5 & 8\end{array}\right]^{T}$, and then open the sliding mode controller when $t=5(\mathrm{sec})$. Figure 2 shows the results of numerical simulation [10-12]. From Figure 2, after opening system (20), they respectively stabilize the points within little time, besides, the corresponding switching manifolds of each points soon converge to zero. 


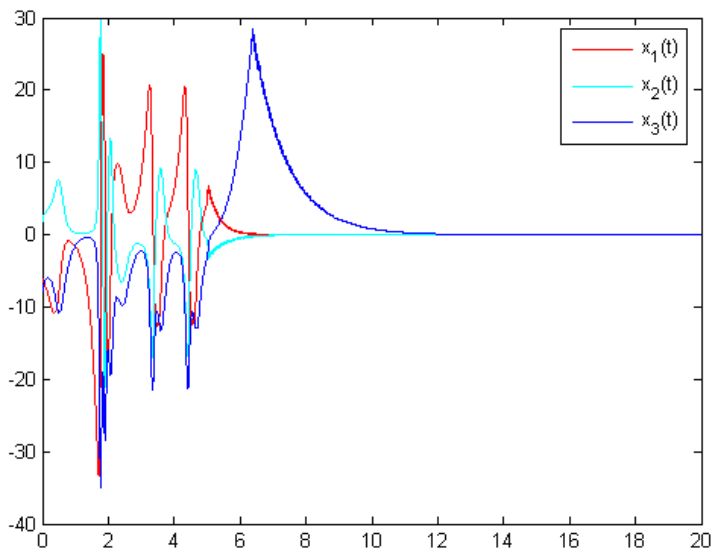

(a) The response curve when system(20) stabilizes at $\overline{\boldsymbol{X}}_{0}$ point

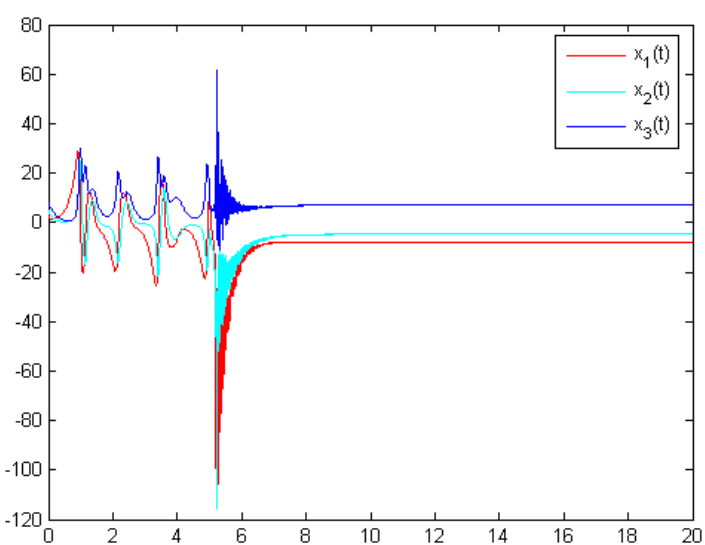

(c) The response curve when system (20)

$$
\text { stabilizes at } \overline{\boldsymbol{X}}_{1} \text { point }
$$

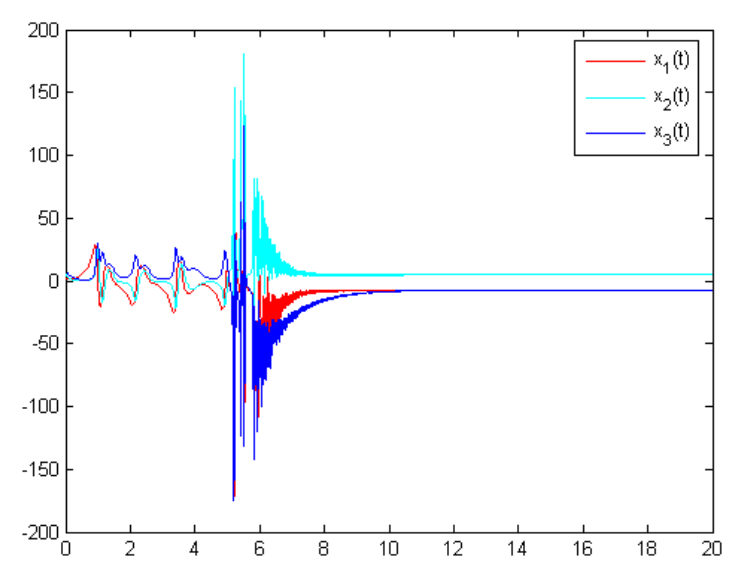

(e) The response curve when system(20) stabilizes at $\overline{\boldsymbol{X}}_{2}$ point

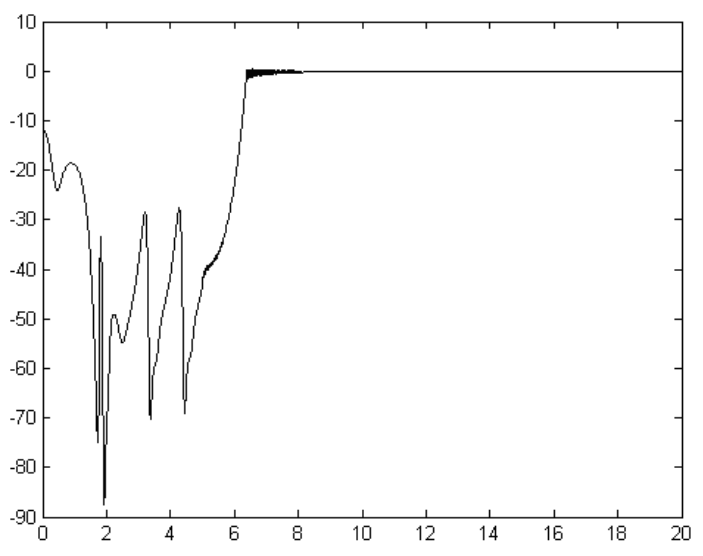

(b) The corresponding response curve of $\mathrm{S}$ when stabilizing at $\overline{\boldsymbol{X}}_{0}$ point

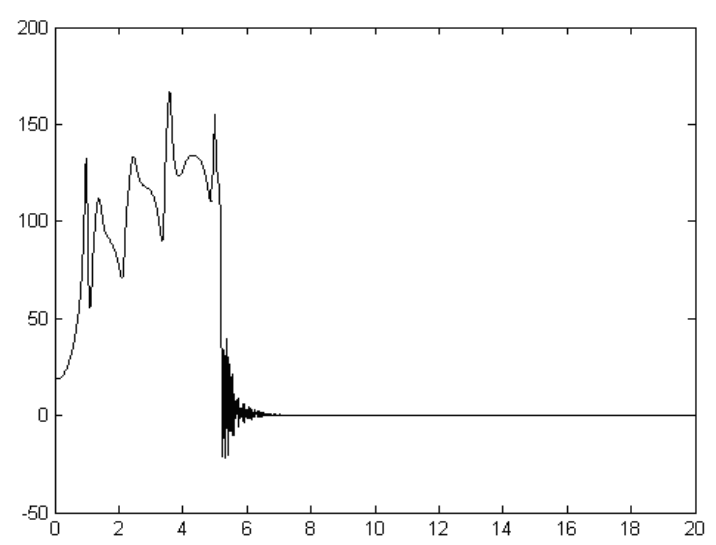

(d) The corresponding response curve of $\mathrm{S}$

when stabilizing at $\overline{\boldsymbol{X}}_{1}$ point

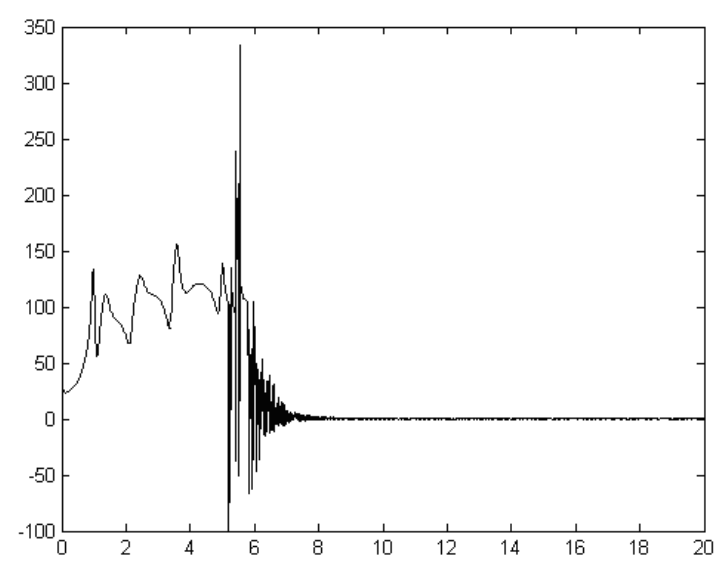

(f) The corresponding response curve of $\mathrm{S}$ when stabilizing at $\overline{\boldsymbol{X}}_{2}$ point 


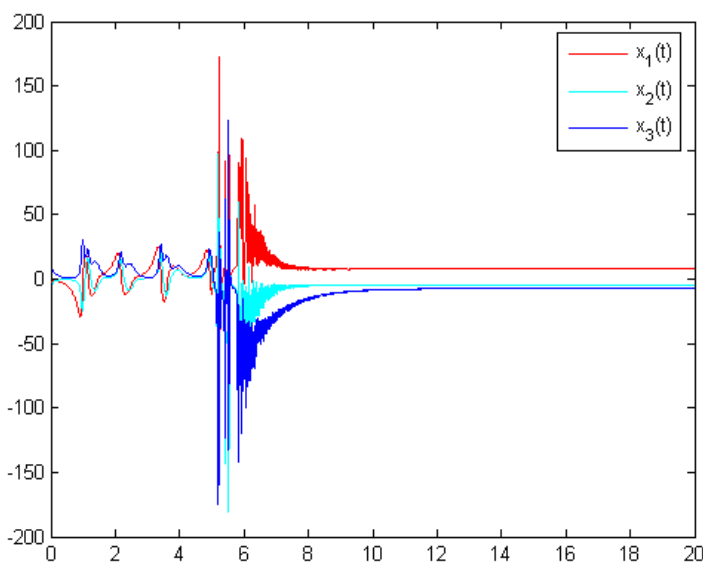

(g) The response curve when system(20) stabilizes at $\overline{\boldsymbol{X}}_{3}$ point

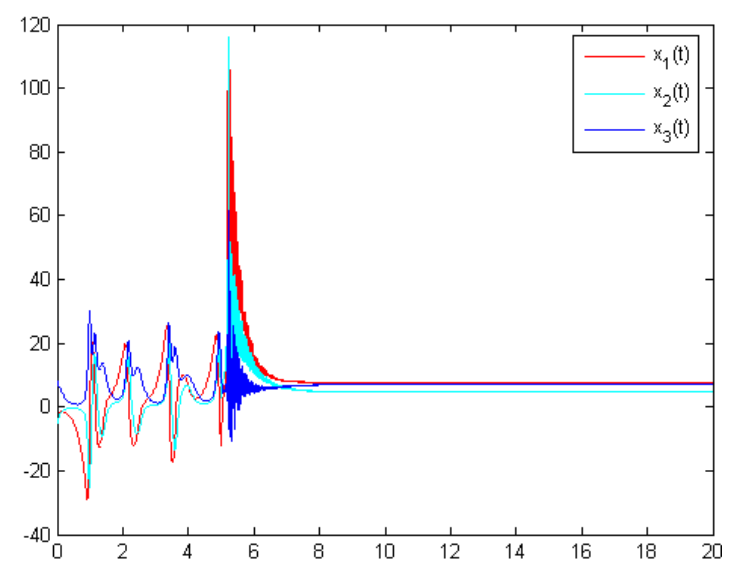

(i) The response curve when system (20) stabilizes at $\overline{\boldsymbol{X}}_{4}$ point

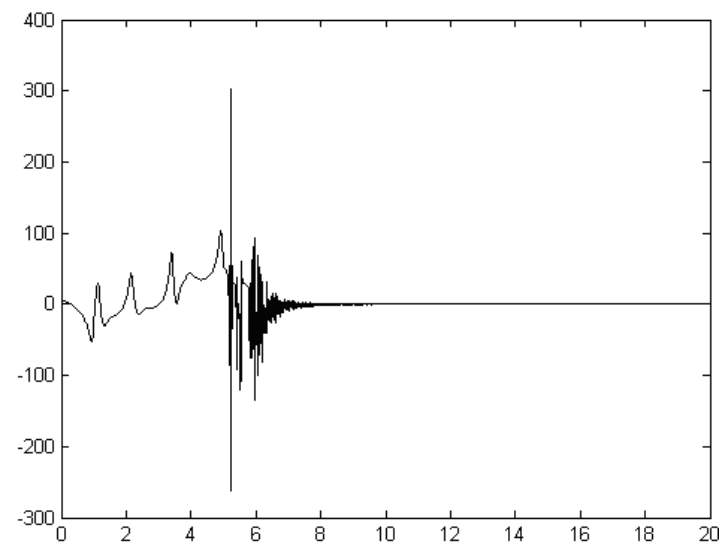

(h) The corresponding response curve of $\mathrm{S}$ when stabilizing at $\overline{\boldsymbol{X}}_{3}$ point

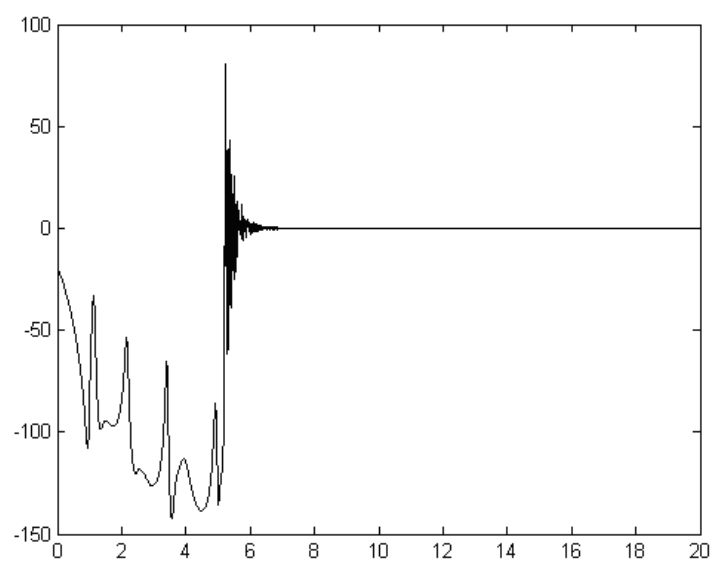

(j) The corresponding response curve of $S$ when stabilizing at $\overline{\boldsymbol{X}}_{4}$ point

Figure 2. The Response Curve of System When Stabilizing at Each Equilibrium Point and Its Corresponding Response Curve of S

\subsection{The control of chaotic attractors with four vortexes}

Make controller parameter $a=0.4, b=12, c=5$, and choose

$$
\boldsymbol{C}=\left[\begin{array}{lll}
1 & 0 & 0 \\
0 & 1 & 0 \\
0 & 0 & 1
\end{array}\right],
$$

When $\boldsymbol{C B} \neq 0$. Use pole assignment method to choose

$$
\boldsymbol{K}=\left[\begin{array}{lll}
-3.4 & 0 & 0 \\
0 & 10 & 0 \\
0 & 0 & 4
\end{array}\right]
$$


Make sure that all the real parts of eigenvalue in matrix $\boldsymbol{A}+\boldsymbol{B} \boldsymbol{K}$ are negative numbers. Then take constant $\lambda=1.6$ and meet the condition of $\lambda>1$.

$$
\begin{gathered}
\boldsymbol{S}=\boldsymbol{C} \boldsymbol{E}-\int_{0}^{t}(\boldsymbol{C A}+\boldsymbol{C B} \boldsymbol{K}) \boldsymbol{E}(\tau) d \tau \Rightarrow\left\{\begin{array}{l}
s_{1}=E_{1}(\tau)-\int_{0}^{t}\left[\begin{array}{lll}
-3 & 0 & 0
\end{array}\right] \boldsymbol{E}(\tau) d \tau \\
s_{2}=E_{2}(\tau)-\int_{0}^{t}\left[\begin{array}{lll}
0 & -2 & 0
\end{array}\right] \boldsymbol{E}(\tau) d \tau . \\
s_{3}=E_{3}(\tau)-\int_{0}^{t}\left[\begin{array}{lll}
0 & 0 & -1
\end{array}\right] \boldsymbol{E}(\tau) d \tau
\end{array}\right. \\
U=-\lambda(\boldsymbol{C B})^{-1}\|\boldsymbol{C B}\|(\|\boldsymbol{K} \boldsymbol{E}\|+\|\boldsymbol{F}\|) \operatorname{sign}(\boldsymbol{S}) \Rightarrow\left\{\begin{array}{l}
u_{1}=-1.6\left(\left\|\left[\begin{array}{lll}
-3.4 & 0 & 0
\end{array}\right] \boldsymbol{E}\right\|+\left\|\boldsymbol{F}^{\prime}\right\|\right) \operatorname{sign}\left(s_{1}\right) \\
u_{2}=-1.6\left(\left\|\left[\begin{array}{lll}
0 & 10 & 0
\end{array}\right] \boldsymbol{E}\right\|+\left\|\boldsymbol{F}^{\prime}\right\|\right) \operatorname{sign}\left(s_{2}\right) \\
u_{3}=-1.6\left(\left\|\left[\begin{array}{lll}
0 & 0 & 4
\end{array}\right] \boldsymbol{E}\right\|+\left\|\boldsymbol{F}^{\prime}\right\|\right) \operatorname{sign}\left(s_{3}\right)
\end{array}\right.
\end{gathered}
$$

Adopt Runge-Kutta's method to solve system (20) and select time step $\tau=0.005$ (sec). The equilibrium of system (18) is $\overline{\boldsymbol{X}}_{0}=\left[\begin{array}{lll}0 & 0 & 0\end{array}\right]^{\mathrm{T}}, \overline{\boldsymbol{X}}_{1}=\left[\begin{array}{lll}-7.746 & -1.4142 & 2.1909\end{array}\right]^{\mathrm{T}}$, $\overline{\boldsymbol{X}}_{2}=\left[\begin{array}{lll}-7.746 & 1.4142 & -2.1909\end{array}\right]^{\mathrm{T}} \quad, \quad \overline{\boldsymbol{X}}_{3}=\left[\begin{array}{lll}7.746 & -1.4142 & -2.1909\end{array}\right]^{\mathrm{T}}$ $\overline{\boldsymbol{X}}_{4}=\left[\begin{array}{lll}7.746 & 1.4142 & 2.1909\end{array}\right]^{\mathrm{T}}$. The initial value of system is $\boldsymbol{X}_{00}=\boldsymbol{X}_{01}=\boldsymbol{X}_{02}=\boldsymbol{X}_{03}=\boldsymbol{X}_{04}=\left[\begin{array}{llll}-6 & 1.7 & -8\end{array}\right]^{\mathrm{T}}$, then open the sliding mode controller when $t=5(\mathrm{sec})$. Figure 3 shows the results of numerical simulation. From Figure 3, after opening system (20), they respectively stabilize at the points $\overline{\boldsymbol{X}}_{0}, \overline{\boldsymbol{X}}_{1}, \overline{\boldsymbol{X}}_{2}$, $\overline{\boldsymbol{X}}_{3}$ and $\overline{\boldsymbol{X}}_{4}$ within little time, besides, the corresponding switching manifolds $\boldsymbol{S}_{\text {of each }}$ points soon converge to zero.

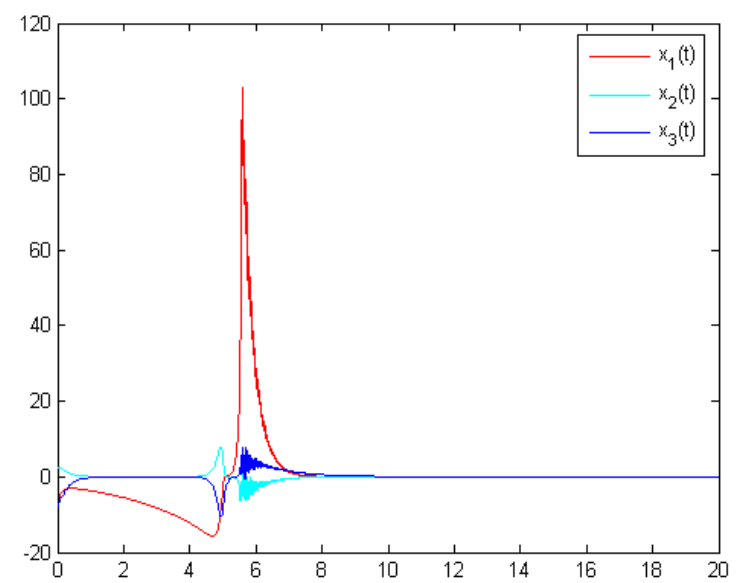

(a) The response curve when system(20) stabilizes at $\overline{\boldsymbol{X}}_{0}$ point

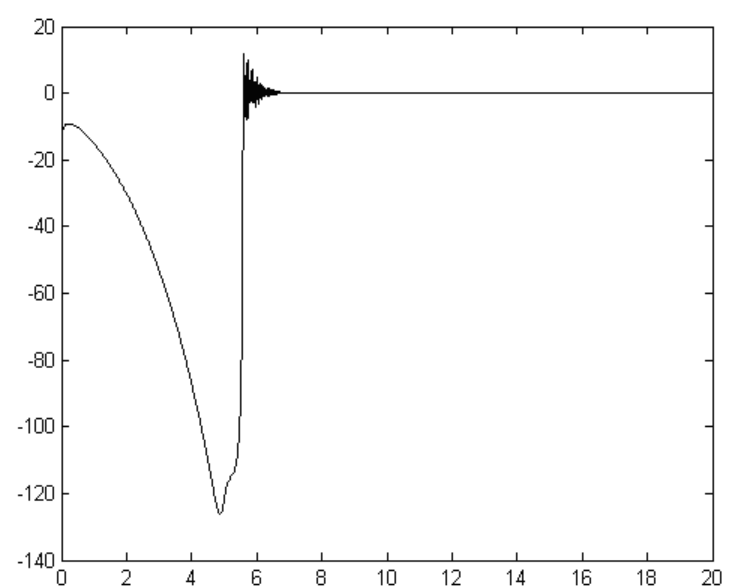

(b) The corresponding response curve of $S$ when stabilizing at $\overline{\boldsymbol{X}}_{0}$ point 


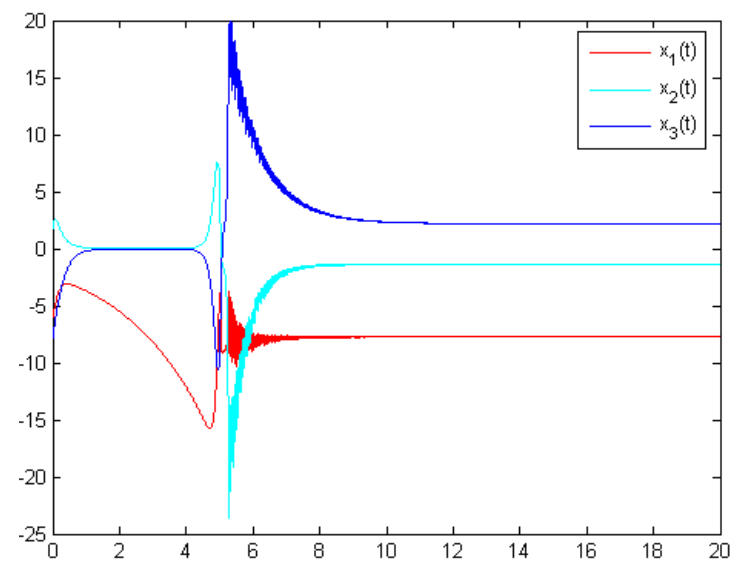

(c) The response curve when system (20) stabilizes at $\overline{\boldsymbol{X}}_{1}$ point

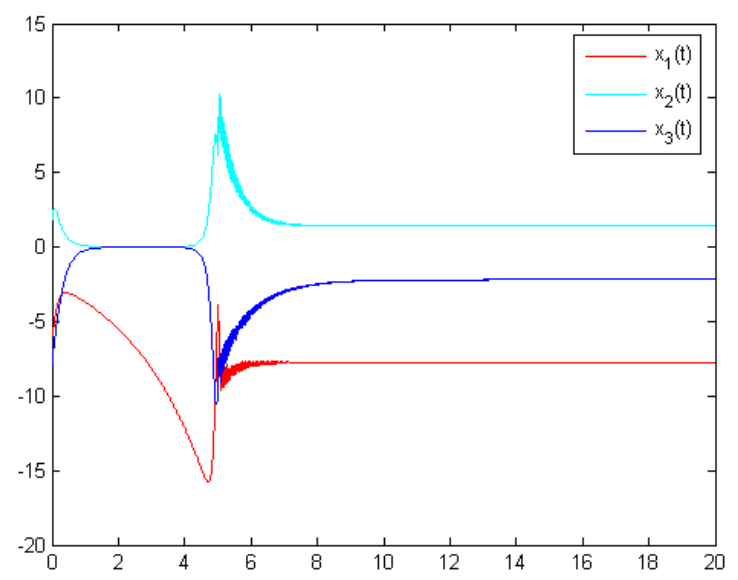

(e) The response curve when system (20) stabilizes at $\overline{\boldsymbol{X}}_{2}$ point

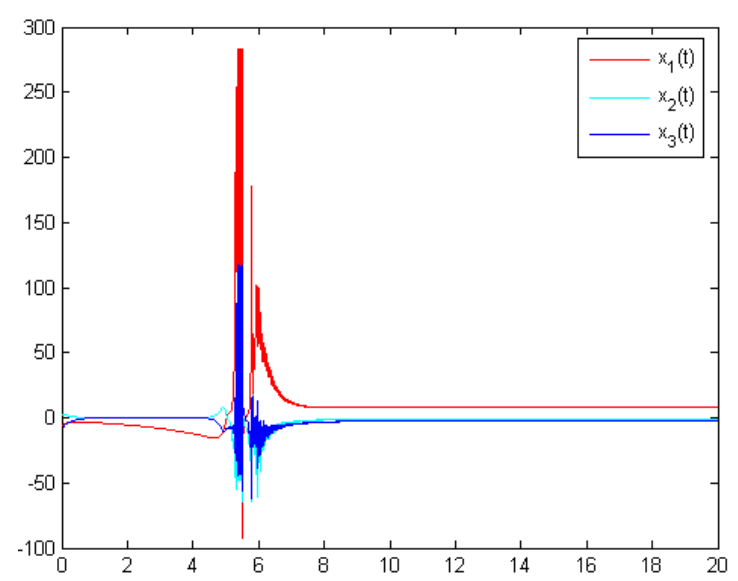

(g) The response curve when system (20) stabilizes atpoint $\overline{\boldsymbol{X}}_{3}$

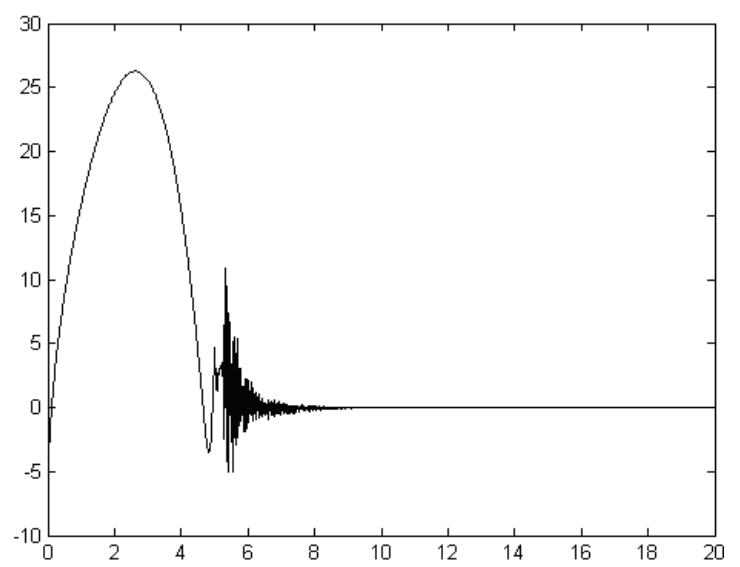

(d) The corresponding response curve of $\mathrm{S}$ when stabilizing at $\overline{\boldsymbol{X}}_{1}$ point

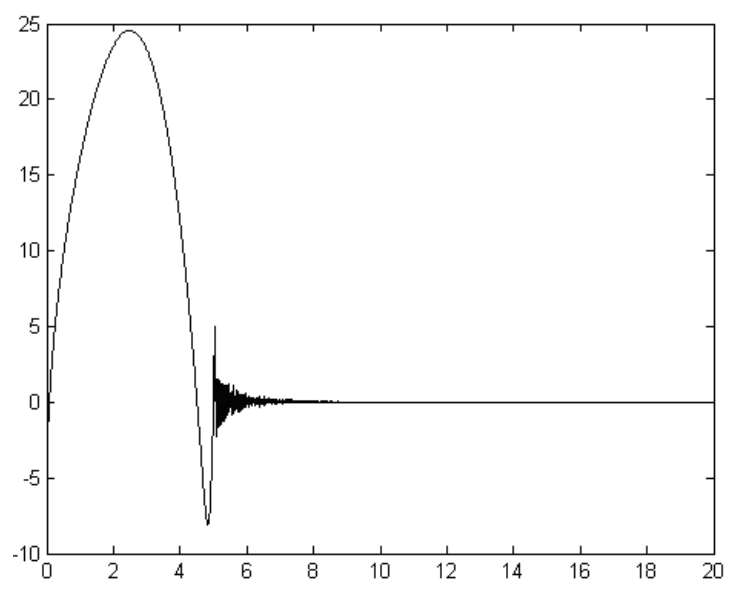

(f) The corresponding response curve of $\mathrm{S}$ when stabilizing at $\overline{\boldsymbol{X}}_{2}$ point

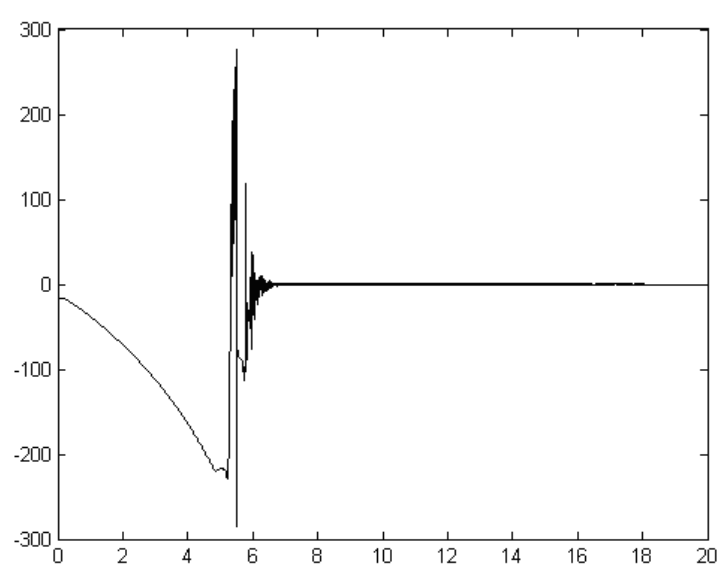

(h) The corresponding response curve of $\mathrm{S}$ when stabilizing at $\overline{\boldsymbol{X}}_{3}$ point 


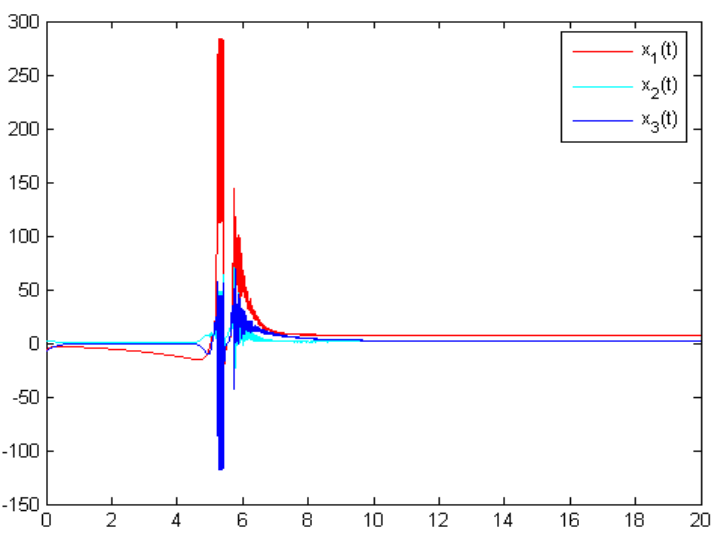

(i) The response curve when system (20) stabilizes atpoint $\overline{\boldsymbol{X}}_{4}$

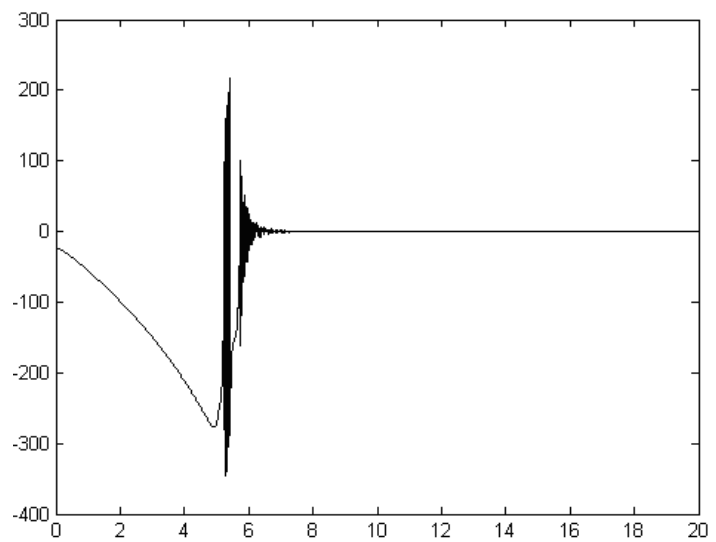

(j) The corresponding response curve of $\mathrm{S}$ when stabilizing at $\overline{\boldsymbol{X}}_{4}$ sw point

Figure 3. The Response Curve of System When Stabilizing at Each Equilibrium Point and Its Corresponding Response Curve of S

\section{Conclusion}

This thesis makes a research about the Sliding Mode Control of a novel chaotic system. Taking advantage of PI switching method, it designs a kind of sliding mode controller and theoretically proves its global stability. The system enters into the state of sliding mode from the beginning so that it can ensure comparatively high robustness. The realization of Sliding Mode Control is extremely simple, what's more, the design of controller and sliding mode surface of each area is only related to the state of area itself, not involving the state information of other areas, in this way can it achieve the decentralized control of system. Numerical simulation experiment shows that: the controlled new chaotic system can stabilize at the appointed equilibrium point with little time, and its corresponding switching manifold $\mathrm{S}$ converges to zero only a little while, which further proves that the availability of this kind of controller.

\section{References}

[1] M. S. Willsey, K. M. Cuomo and A. V. Oppenheim, "Quasi-Orthogonal Wideband Radar Waveforms Based on Chaotic Systems, IEEE Transactions on Aerospace and Electronic Systems, vol. 47, no. 3, pp. 1974-1984, 2011.

[2] E. Ott, C. Grebogi and J. A. Yorke, "Controlling chaos", Physical Review Letters, vol. 64, no. 11, (1990), pp 1196-1199.

[3] Y. Liu, S. Zhao and J. Lu, "A New Fuzzy Impulsive Control of Chaotic Systems Based on T-S Fuzzy Model”, IEEE Transactions on Fuzzy Systems, vol. 19, no. 2, (2011), pp. 393-398.

[4] Y. Liang and H. J. Marquez, "Robust Gain Scheduling Synchronization Method for Quadratic Chaotic Systems With Channel Time Delay", IEEE Transactions on Circuits and Systems, vol. 56, no. 3, (2009), pp. 604-615.

[5] F. Pareschi, G. Setti and R. Rovatti, "Implementation and Testing of High-Speed CMOS True Random Number Generators Based on Chaotic Systems", IEEE Transactions on Circuits and Systems, vol. 57, no. 12, (2010), pp. 3124-3137.

[6] T. H. Yeap and N. U. Ahmed, "Feedback control of chaotic systems", Dynamics and Control, vol. 4, no. 1, (1994), pp. 97-114.

[7] J. A. Gallegos, "Nonlinear regulation of a Lorenz system by feedback linearization techniques", Dynamics 
and Control, vol. 4, no. 3, (1994), pp. 277-298.

[8] C. C. Fuh and P. C. Tung, "Controlling chaos using differential geometric method", Physical Review Letters, vol. 75, no. 16, (1995), pp. 2952-2955.

[9] Y. Zeng and S. N. Singh, "Adaptive control of chaos in Lorenz system", Dynamics and Control, vol. 7, no. 2, (1997), pp. 143-154.

[10] M. D. Bernardo, "An adaptive approach to the control and synchronization of continuous-time chaotic systems", International Journal of Bifurcation and Chaos, vol. 6, no. 3, (1996), pp. 557-568.

[11] M. Feki, "An adaptive feedback control of linearizable chaotic systems", Chaos, Solitons \& Fractals, vol. 15, no. 5, (2003), pp. 883-890.

[12] C. W. Wu, T. Yang and L. O. Chua, "On adaptive synchronization and control of nonlinear dynamical systems”, International Journal of Bifurcation and Chaos, vol. 6, no. 3, (1996), pp. 455-471.

\section{Author}

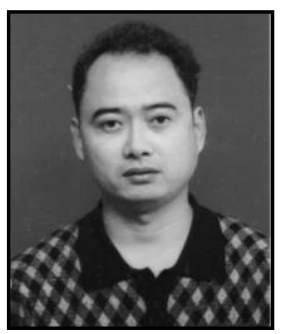

\section{Xuexiang Li}

He work in Department of Information Engineering, ZiBo Vocational Institute, China. Research area is computer application technology, and his educational level is Master of Software Engineering. 